3 Drought is one of the prime abiotic stresses in the world. Now, amongst the new

\section{Molecular Predicting Drought Tolerance in Maize Inbred Lines by Machine} Learning Approaches technologies available for speed up the releasing of new drought tolerance genotypes, there is an emanate discipline called machine learning. The study presents Machine Learning for identification, classification and prediction of drought tolerance maize inbred lines based on SSR genetic markers datasets generated from PCR reactions. A total of 356 SSR reproducible fragment alleles were detected across the 71 polymorphic SSR loci. A dataset of 12 inbred lines with these fragments prepared as attributes and was imported into RapidMiner software. After removal of duplicates, useless and correlated features, 311 feature attributes were polymorphic, ranging in size from 1500 to $3500 \mathrm{bp}$. The most important attribute fragment alleles in different attribute weighting selected. Ten datasets created using attribute selection (weighting) algorithms. Different classification algorithms were applied on datasets. These can be used to identify groups of alleles with similar patterns of expression, and are able to create some models that have been applied successfully in the prediction, classification and pattern recognition in drought stress. Some unsupervised models were able to differentiate tolerant inbred lines from susceptible. Four unsupervised models were able to produce the different decision trees with root and leaves. The most important attribute alleles almost in all of models were phi033a3, bnlg1347a1 and bnlg172a2 respectively, that can help to identify tolerant maize inbred lines with high precision.

Keywords: Prediction, Drought Tolerance, Abiotic Stress, Machine Learning, Classification

Abbreviation: Fcdb (final cleaned database), SVM (Support Vector Machine), PCA (Principle Component Analysis), EM (Expectation Maximization) 


\section{Introduction}

Drought is the main cause of reduced yields in cereal crops (Edmeades et al., 2000), after low soil fertility, the second most important cause of yield loss for maize (Dariano et al., 2016). It is a major limitation in maize production, particularly under climatic changes (Lobell et al., 2014), leading to a grain yield reduction of $25-30 \%$, even without any harvests during extremely severe drought events (Khazaei et al., 2013; Singh et al., 2016). Drought has greater effect despite cultivar and agronomic management improvements. Climatic warming is expected to further enhance the harmful impact of droughts; potentially leading to a significant decrease in maize yields (Ribaut et al., 2009; Lobell et al., 2014). Thus, research on maize drought tolerance and its mechanisms remain hotspot under the pressure of increasing environmental conditions that reflect the impact of human activities on the environment that leads to the appearance of environmental problems. (Lobell et al., 2014; Khazaei et al. 2013; Ribaut et al., 2009). Drought has even been thought of as a "cancer" to plants, owing to its complexity and destructiveness. Hence, there is enormous interest in and demand for improving maize drought tolerance through biotechnology (Wang et al., 2016).

Maize respond drought stress at the physiological, biochemical, and molecular levels to adapt to changing environmental conditions (Jaglo-Ottosen et al., 1998; Shinozaki et al., 2003; Xiong et al., 2002). The identification and use of molecular markers to assist in selection of quantitative trait loci, genome-wide selection, and association mapping have become common areas of research. In the future, the destructive impact of drought may grow, as the spectre of climate change becomes a reality. With the development of PCR-based DNA markers such as RAPD (Raibut 2009) SSR (Ercisli et al., 2011), AFLPs (Pafundo et al., 2005) and single nucleotide polymorphism (Reale et al. 2006), marker technology today offers a palette of powerful tools to analysis the plant genome. (Tardieu 2012; Wang et al. 2011; Yadav et al., 2011). They have enabled the identification of genes and genome associated with the expression of qualitative and quantitative traits and has led to a better understanding of the complex genome of various plants (Shinozaki and YamaguchiShinozaki, 2007; Tao et al., 2011), besides helping in identifying the desired species at any growth stage of the plant. Despite numerous published reports of molecular markers for drought-related traits, practical applications of such results in maize 
63 improvement are scarce (Benesova et al., 2012) and results are not completely

64 satisfying and more research on methodologies is needed (Ornella et al., 2012).

Machine learning is an emerging inherently multidisciplinary approach to data analysis with a revolutionary impact on a variety of areas and refers to a group of computerized modelling approaches that can learn patterns from the data to make automatic decisions without programming obvious rules. (Dror et al., 2005; Hajiloo et al., 2013; Hepworth et al., 2012, Hor et al., 2013; Mutka and Bart , 2015; Smith et al., 2013; Tran, 2014; Yan et al., 2013). The main idea of machine learning is to effectively utilize experiences to discover main underlying structures, similarities, or dissimilarities present in data to explain or classify a new experience properly. A key ability of machine learning tools, in its most fundamental form, is their ability to generalize complex patterns and making intelligent decisions from data. (Sing et al., 2016; Forghani and Yazdi, 2014; Ma et al., 2012). Machine learning will also enhance our understanding of pathogen-plant interactions as well as the interaction of plants with other stresses (Kuska et al. 2015; Sing et al. 2016; Romer et al., 2012). One of the major advantages of machine-learning approaches for plant breeders and biologists is the opportunity to search datasets to discover patterns and govern discovery by simultaneously looking at a combination of factors instead of analysing each feature individually (Mutka and Bart, 2015; Sing et al., 2016).

Due to their high generalization capabilities and distribution-free properties they are presented as a valuable alternative to traditional statistical techniques applied in maize breeding, even the more recently introduced linear mixed models (Maenhout et al., 2010; Maenhout et al., 2007; Ornella, Cervigni and Tapia, 2012).

In this study, using polymorphic SSR loci, molecular analysis of the selected set of inbred lines carried out. After detecting SSR reproducible fragment alleles, datasets were created with different attribute weighting methods. Different unsupervised and supervised classification algorithms run on datasets. Machine learning models differentiate tolerant maize inbred lines from susceptible. The main objective of this study is to develop machine-learning models in the field of plant stress phenotyping in identification, classification, quantification and prediction.

93 Understanding the genetic basis of drought tolerance of maize with major attribute alleles may facilitate efforts to improve drought tolerant inbred lines. 
96

97

98

99

100

101

102

103

104

105

106

107

108

109

110

111

112

113

114

115

116

117

118

119

120

121

122

123

124

125

126

127

\section{Materials and Methods}

A selected set of 12 inbred lines with distinct tolerance and susceptibility responses to drought stress at the flowering stage in maize were analyzed in this study. These include four DTPY lines (DTPY65, DTPY108, DTPY116 and DTPY194), three DTPW lines (DTPW51, DTPW105, DTPW183 and DTPW195) besides Ac7643, Ac7729, CM139 and CM140. Genomic DNA from each of genotypes was extracted from a bulk of 10-15 plants, using a modified CTAB method (Saghi-Maroof et al., 1994) with suitable modifications. Primers for SSR loci were custom synthesized through Research Genetics Inc., USA or Sigma Aldrich, UK, based on forward and reverse primer sequence information available in public domain (MaizeDB; http://www.agron.missouri.edu).

Molecular analysis of the selected set of inbred lines was carried out using 71 polymorphic SSR loci covering various bin (chromosome) locations (Table 1). In addition, SSR markers located at specific bin locations with potential utility for QTL mapping of drought tolerance in maize were utilized in the study (Table 1). PCR reactions were carried out in $10 \mu \mathrm{l}$ reaction volume containing of $10 \times$ PCR buffer (10 $\mathrm{mM}$ Tris- $\mathrm{HCl} \mathrm{pH} 8.3,50 \mathrm{mM} \mathrm{KCl}), 1.5 \mathrm{mM} \mathrm{MgCl}_{2}, 0.2 \mathrm{mM}$ each dNTPs, $5 \mathrm{pmol}$ of each forward and reverse primer, $0.5 \mathrm{U}$ of Taq DNA polymerase (NEB), and $5 \mathrm{ng}$ of genomic DNA. Reactions were carried out in GenAmp PCR system 9700 (Applied Biosystems, USA) thermal cycler using the following temperature profile: an initial denaturation of $5 \mathrm{~min}$ at $94^{\circ} \mathrm{C}$ followed by 35 cycles of $45 \mathrm{~s}$ at $94^{\circ} \mathrm{C}, 45 \mathrm{~s}$ at $55^{\circ} \mathrm{C}$ and $1 \mathrm{~min}$ at $72^{\circ} \mathrm{C}$, then a final extension of $5 \mathrm{~min}$ at $72^{\circ} \mathrm{C}$. Amplification products were resolved on 3\% metaphor agarose gels using a horizontal gel electrophoresis unit (CBS Scientific, USA). The DNA fragments were then visualized under UVtransilluminator and documented using ALPHA IMAGER gel documentation system (Alpha Innotech, USA) which was stored for further scoring and permanent records. The fragment alleles were visually scored as present (1) or absent (0).

3 Allele designations were made for the amplification products for each SSR locus was determined based on the positions of the bands relative to the $100 \mathrm{bp}$ molecular-weight ladder (Fig. 1). A total of 356 SSR reproducible fragment alleles were detected across the 71 polymorphic SSR loci. A dataset of 12 inbred lines with these fragments prepared as attributes and was imported into RapidMiner software 
128 [RapidMiner 5.2, Rapid-I GmbH, Stochumer Str. 475, 44227 Dortmund, Germany].

129 Then, the steps detailed below were applied to this dataset.

\section{Data cleaning}

132 Useless attributes were removed from the dataset. Nominal attributes were 133 regarded as useless when the most frequent values were contained in more or less

134 than nominal useless above or below per cent of all examples. After cleaning, this 135 database was labelled the final cleaned database (FCdb).

\section{Attribute Weighting}

137 To identify the most important allele attributes and to find the possible patterns 138 in features that contribute to different maize inbred lines, 10 different algorithms of 139 attribute weightings including Information gain, Information Gain ratio, Rule, Deviation, 140 Chi squared statistic, Gini index, Uncertainty, Relief, SVM and PCA were applied to 141 the FCdb. Weight by Information gain operator calculated the relevance of a feature 142 by computing the information gain in class distribution. Weight by Information Gain 143 ratio calculated the relevance of a feature by computing the information gain ratio for 144 the class distribution. Weight by Rule calculated the relevance of a feature by 145 computing the error rate of a OneR Model on the example set without this feature. 146 Weight Deviation operator created weights from the standard deviations of all 147 attributes. The values were normalised by the average, the minimum, or the maximum 148 of the attribute. Weight by Chi squared statistic operator calculated the relevance of a 149 feature by computing, for each attribute of the input example set, the value of the chi150 squared statistic with respect to the class attribute. Weight by Gini index operator 151 calculated the relevance of an attribute by computing the Gini index of the class 152 distribution, if the given example set would have been split according to the feature. 153 Weight by Uncertainty operator calculated the relevance of an attribute by measuring 154 the symmetrical uncertainty with respect to the class. Weight by Relief measured the 155 relevance of features by sampling examples and comparing the value of the current 156 feature for the nearest example of the same and of a different class. This version also 157 worked for multiple classes and regression data sets. The resulting weights were 158 normalised into the interval between 0 and 1. Weight by SVM operator used the 
159 coefficients of the normal vector of a linear SVM as feature weights. Weight by PCA

160 operator used the factors of the first of the principal components as feature weights.

\section{Attribute selection}

After attribute weighting models were run on the dataset, each alleles attribute

164 (feature) gained a value between 0 and 1, which revealed the importance of that 165 attribute with regards to a target attribute (susceptible-tolerance cultivar). All variables

166 with weights higher than 0.50 were selected and 10 new datasets created and were 167 named according to their attribute weighting models (Information gain, Information 168 gain ratio, Rule, Deviation, Chi Squared, Gini index, Uncertainty, Relief, SVM and $169 P C A$. These newly formed datasets were used to join with subsequent models 170 (supervised and unsupervised). Each model of supervised or unsupervised clustering 171 were performed 11 times; the first time it ran on the main dataset (FCdb) and then on 172 the 10 newly formed datasets from attribute weighting and selection.

\section{Unsupervised clustering algorithms}

174 Ten newly created datasets generated as the outcomes of different attribute 175 weighing algorithms along with FCdb were applied to K-Means, K-Medoids, Support 176 Vector Clustering (SVC) and Expectation Maximization (EM) clustering algorithms. K177 Means uses kernels to estimate the distance between objects and clusters. Because 178 of the nature of kernels, it is necessary to sum over all elements of a cluster to 179 calculate one distance. K-Medoids represents an implementation of k-Medoids. This 180 operator will create a cluster attribute if it is not yet present. SVC represents 181 implementations of Support Vector algorithm which will create a cluster attribute if not 182 present yet. EM represents an implementation of the EM-algorithm.

\section{Supervised Classification}

184 Three classes of supervised classification (Decision Trees, SVM and Baysian 185 models) applied as follows. To calculate the accuracy of each model, 10-fold cross 186 validation is used to train and test models on all patterns. To perform cross validation, 187 all the records were randomly divided into five parts; four sets were used for training 188 and the 5th one for testing. The process was repeated five times and the accuracy for 189 true, false and total accuracy calculated. The final accuracy is the average of the 190 accuracy in all five tests. 


\section{Decision Trees}

192 Six tree induction models including Decision Tree, Decision Tree Parallel,

193 Decision Stump, Random Tree, ID3 Numerical and Random Forest were run on the

194 main dataset (FCdb). Each tree induction model ran with the following four different

195 criteria: Gain Ratio, Information Gain, Gini Index and Accuracy. In addition, a weight-

196 based parallel decision tree model, which learns a pruned decision tree based on an

197 arbitrary feature relevance test (attribute weighting scheme as inner operator), was

198 run with 13 different weighing criteria (SVM, Gini Index, Uncertainty, PCA, Chi

199 Squared, Rule, Relief, Information Gain, Information Gain Ratio, Deviation,

200 Correlation, Value Average, and Tree Importance). The accuracy of each tree

201 computed based on the previous explanation.

\section{Support Vector Machine Approach}

203 Support Vector Machines (SVMs) are popular and powerful techniques for 204 supervised data classification and prediction; so SVM, LibSVM, SVM Linear and 205 SVME used here to implement different models to predict maize cultivars based on 206 Susceptible-Tolerance features. Briefly, main database (FCdb) transformed to SVM 207 format and scaled by grid search (to avoid attributes in greater numeric ranges 208 dominating those in smaller numeric ranges) and to find the optimal values for 209 operator parameters. To prevent overfitting problems, 5-fold cross validation applied.

210 Dataset divided into 5 parts and 4 parts used as training set and the last part as 211 testing set. The procedure repeated for 10 different testing sets and the average of 212 accuracy computed. RBF kernel that nonlinearly maps samples into a higher 213 dimensional space and can handle the case when the relation between class labels 214 and attributes is nonlinear used to run the model. Other kernels such as linear, poly, 215 sigmoid and pre-computed were also applied to the dataset to find the best accuracy.

\section{Naïve Bayes}

217 Naïve Bayes based on Bayes conditional probability rule was used for 218 performing classification tasks (Wang and Tseng 2013). Naïve Bayes assumes the 219 predictors are statistically independent which makes it an effective classification tool 220 that is easy to interpret. Two models, Naïve base (returns classification model using 221 estimated normal distributions) and Naïve base kernel (returns classification model 
222 using estimated kernel densities) used and the model accuracy in predicting the right

223 resistance - susceptible computed as stated before.

\section{Results}

225 SSR alleles differing by several repeat units can often be distinguished on 226 agarose gels. High level of allelic variation, making SSR valuable as genetic 227 attributes (Fig. 1). SSR is used for understanding of the evolutionary genetics and 228 sequences controlling traits of economic interest of maize (Xu et al., 2009).

229 As mentioned in Materials and Methods, the initial dataset contained 12 maize 230 inbred lines with 356 SSR fragment attributes. Following removal of duplicates, 231 useless attributes, and correlated features (data cleaning) 311 features remained, 232 meaning these fragment attributes were polymorphic, ranging in size from 1500 to $2333500 \mathrm{bp}$.

\section{Attribute weighting}

235 The number of attributes gained weights higher than 0.5 in each weighting 236 model were as follows: Relief 168, Rule 168, Deviation 147, SVM 134, PCA 93, Info 237 gain ratio 20, Uncertainty 20, Chi squared 9, Gini index 8 and Info Gain 8 (Table 2). 238 The most important attribute fragment allele in all of models was phi033a3, which

239 weighted equal to 1.0 in all weighting models except to PCA and can help to identify 240 tolerance maize inbred lines from susceptible.

241 In weighting by Deviation operator 46 attribute alleles including bnlg2323a1, 242 bnlg1730a4, phi088a3, bnlg1655a2, mage05a3 and bnlg210a3 weighed equal to 243 1.0. In Weighting by Rule model five attributes, phi033a3, bnlg172a3, phi078a2, 244 bnlg381a3 and bnlg172a2; in SVM model three attributes, phi033a3, bnlg1347a1 245 and bnlg1347a2; and in PCA model five attributes, bnlg1138a1, bnlg18a3, phi102a3 246 and phi102a2 weighted equal to 1.0. These attributes were the most important 247 attributes selected when these models applied on dataset of correlated removed 248 features. Five of ten attribute weighting models including Uncertainty, Info Gain 249 Ratio, Gini Index, Information Gain and Chi Squared selected the following attribute 250 alleles: phi033a3, bnlg172a2, bnlg1347a1, bnlg1347a2, umc1572a4, bnlg172a3 and 251 bnlg381a3 weighted more than 0.7. In Weighting by Releif model only phi033a3 252 attribute allele weighted equal to 1.0. These attributes were the most important 253 attributes selected when these models applied on dataset of correlated removed 
254 features (Table3). Almost all of these attributes are the main branches of decision

255 trees (Fig. 3 and Fig. 4).

\section{Unsupervised Clustering Algorithms}

257 Three different unsupervised clustering algorithms (K-Means, K-Medoids and 258 SVC) were applied on ten datasets created using attribute selection (weighting) 259 algorithms. Some models, such as the application of the SVC algorithm on all 260 datasets were able to differentiate tolerant inbred lines from susceptible. In this 261 algorithm all tolerant inbred lines predicted as correct class of tolerance (Fig. 2-A). 262 Application of the K-Means to SVM, PCA and Deviation databases was able to assign 263 respectively $66.7 \%, 33.3 \%$ and $50 \%$ drought tolerance inbred lines into its correct 264 class while application of $K$ - Medoids to same database was able to assign 265 respectively 50\%, 83.3\% (Fig. 2-C) and 100\% (Fig. 2-B) drought tolerance inbred 266 lines into its correct class (Table 4). Combination of $K$-Medoids clustering method 267 with Deviation attribute weighting selected the right classes of drought tolerance 268 inbred lines (Fig. 2-B) while Combination of K-Means clustering method with PCA 269 attribute weighting selected the right classes of drought susceptible inbred lines (Fig.

270 2-D).

\section{Supervised Classification}

\section{Decision Trees}

273 From six induction models Random Tree and ID3Numerical were not able to 274 produce decision trees. Decision Stump, Decision Tree, Decision Parallel and 275 Random Forest Tree with 4 from 13 different criteria; gain ratio, information gain, gini 276 index and accuracy (run on 11 datasets) were able to produce the different decision 277 trees with root and leaves (Figure 3 - Figure 5). Decision Stump, Decision Tree and

278 Decision Parallel in 4 criteria Gain Ratio, Information Gain, Gini Index and Accuracy 279 almost on all databases can produce the same simple decision tree (Figure 3), but 280 their overall accuracy is below $60 \%$. In this decision tree phi033a3 attribute fragment 281 allele with the average attribute weighting of 0.92 was the sole fragment allele used to 282 build this tree. If there is phi 033a3 allele, the maize inbred line is tolerance to 283 drought; otherwise, the inbred line is susceptible.

284 Random Forest model with four criteria on Chi Square, Deviation, Information 285 Gain and Gini Index datasets were able to produce different decision trees with 
286 overall accuracy above 50\% (Table5). Decision trees of Random Forest model with

287 Accuracy criterion run on Information Gain dataset (Fig. 4 -A ) and Gini Index Criterion

288 run on Gini Index dataset (Fig. 4 -B) show 100\% overall accuracy and precision.

289 Figure 4 -A shows that bnlg1347a2 attribute fragment allele was the sole fragment

290 allele used to build this tree. If there is bnlg1347a2 allele, the maize inbred line is

291 drought tolerance; otherwise, the inbred line is susceptible. Figure 4 -B shows that

292 bnlg381a3 fragment allele was the sole fragment allele used to build this tree. If there

293 is bnlg381a3 allele, the maize inbred line is tolerance. Random Forest model with Chi

294 Square dataset was able to produce different decision trees with overall accuracy

$29590 \%$ and overall precision 100\% when run with Accuracy (Fig. 5 -A), Gain Ratio (Fig.

2965 -B) and Gini Index criteria (Fig. 5 -C). Random Forest model with Gini Index criterion

297 also produce decision tree (Fig. 5 -D) with overall accuracy 90\% and overall precision

298 100\% when run on Information Gain dataset.

\section{SVM approach}

300 The result of the SVM prediction system based on the 10 fold cross validation 301 sets show that overall accuracies were in the range of $0.0 \%$ to $100 \%$. The overall 302 accuracies and prediction, prediction susceptible and tolerance precision and true 303 susceptible and tolerance recall of 4 algorithms on Chi Square, Gini Index, 304 Information Gain, Relief and Uncertainty databases and LibSVM algorithm on 305 Uncertainty dataset were 100\% (Table 6). The number of support vectors was 10-12 306 and for both susceptible and tolerance cultivars was 5-6 in these algorithm. These 307 results demonstrated the manifest improvement of the prediction accuracies due to 308 the application of generated datasets with Support Vector Machine model.

\section{Naïve Bayes}

311 The overall accuracies and prediction, prediction susceptible and tolerance 312 precision and true susceptible and tolerance recall of Naïve Base and Naïve Kernel 313 run on all databases were 100\% except Deviation, FCdb, PCA and Rule datasets

314 (Table 7). These results show the manifest improvement of the prediction accuracies 315 due to the application of generated datasets with Naïve Base and Naïve Kernel 316 models. Current practices rely heavily on the classical Naïve Bayes algorithm due to 317 its simplicity and robustness. However, results from these algorithms are satisfactory. 


\section{Discussion}

319 The worldwide production of maize (Zea mays L.) is frequently impacted by

320 water scarcity and as a result, increased drought tolerance is a priority target in

321 maize breeding programs (Liu et al., 2013). Understanding the response of a crop to

322 drought is the first step in the breeding of tolerant genotypes (Benesova et al., 2012).

323 It is clear that one of the main objectives of plant breeding is the development of

324 genotypes adapted to stressful environments. Drought resistance is labelled as a 325 'complex trait' mainly when viewed by molecular biologists (Fleury et al. 2010;

326 Prasanna et al. 2009; Ravi et al. 2011). Recent advances in genomics have provided

327 powerful new tools for molecular dissection of drought tolerance in maize during last

328 decades. This platform is capable of expressing hundreds and thousands of drought-

329 responsive genes, which are up or down regulated under dehydration stress

330 according to growth stage, plant organ or even time of day (Blum, 2011). Identify the

331 function of the candidate genes towards drought resistance is difficult. Inter-

332 disciplinary scientists have been trying to understand and dissect the mechanisms of

333 plant tolerance to drought stress using a variety of approaches (Mir et al., 2012). In

334 order to achieve useful results, researchers require methods that consolidate, store

335 and query combinations of structured and unstructured data sets efficiently and

336 effectively. Herein, we combined molecular biology with biological knowledge

337 discovery to determine the most important features contribute to the clustering,

338 classification and prediction of drought tolerance inbred lines from susceptible based

339 on SSR data.

$340 \quad$ The first and most important steps in any data processing task is to verify that 341 your data values are correct or, at the very least, conform to some a set of rules.

342 Data cleaning deals with detecting and removing errors and inconsistencies from

343 data and were used to remove redundancy and co-linearity, useless or duplicated

344 attributes in order to improve the quality of data which results in a smaller database

345 (Ashrafi et al., 2011; Beiki et al., 2012). More than 7\% of the attribute alleles

346 discarded when these algorithms were applied on the original dataset. Each attribute

347 weighting system uses a specific pattern to define the most important SSR fragment

348 alleles. Thus, the results may be different (Baumgartner et al., 2010), as has been

349 highlighted in previous studies (Ashrafi et al., 2011; Beiki et al., 2012; Ebrahimi et al.

350 2011). The results showed that attribute subset selection can be beneficiary both to 
351 processing time and getting more accurate results. This reduces the dimensionality

352 of the data and enables data mining algorithms to predict drought tolerance maize

353 inbred lines faster and more effectively.

354 Attribute selection had an important effect on the classification and 355 identification capability of drought tolerance inbred lines. Attribute weighting and 356 selection methods based on SSR molecular marker data can classify drought 357 tolerance and susceptible inbred lines (Table 2 and Table 3). The phi 033a3, 358 bnlg1347a1, bnlg1347a2, bnlg172a2, bnlg381a3, bnlg381a2, bnlg172a3 and 359 bnlg381a2 SSR fragments were the most important feature to distinguish drought 360 tolerance from susceptible inbred lines, as defined by the entire attribute weighting 361 algorithms (Table 2 and Table 3). These alleles can help to identify tolerance maize 362 inbred lines from susceptible.

363 The goal of unsupervised pattern is to identify small subsets of alleles that 364 display coordinated expression patterns. The unsupervised algorithm figure out the 365 underlying similarities among a set of feature vectors, and to cluster similar vectors 366 together (Ashrafi, Alemzadeh, Ebrahimi, Ebrahimie and Dadkhodaei 2011, Beiki, 367 Saboor and Ebrahimi 2012, Garcia-Gonzalez et al. 2010), while supervised pattern 368 like decision trees are very popular tools for classification (Migliorini et al. 2013, Yun 369 et al. 2014). In unsupervised learning, the machine finds new patterns without 370 relying on prior training examples, usually by using a set of pre-defined rules (Bravo 371 and Medina 2008, El Harchaoui et al. 2013, Handfield et al. 2013, Markovich Gordon 372 et al. 2012). Here we quantify the performance of a given unsupervised clustering 373 algorithm applied to a given molecular marker data in terms of its ability to produce 374 biologically meaningful clusters using a reference set of functional classes. We used 375 three different unsupervised clustering methods (K-Means, K-Medoids and SVC) on 37611 datasets created from SSR fragment allele attributes, which were assigned high 377 weights. The performances of these algorithms varied significantly, usually these 378 algorithms work well when the numbers of classes to be clustered are small. Here 379 we have only two classes, tolerance and susceptible and it is expected that these 380 algorithms are suitable for this condition and there is no need more complex 381 clustering.

382 The results showed that combination of $K$-Medoids clustering method with 383 Deviation attribute weighting was able to assign the drought tolerance inbred lines in 
384 right classes (Fig.2 -B) while Combination of K-Means clustering method with PCA

385 attribute weighting selected the right classes of drought susceptible inbred lines 386 (Fig.2 -D).

387 The main objective of decision analysis is to offer a theoretical representation 388 of choices made in an environment of uncertainty (Berry and Linoff 2004, Senthil 389 Kumar et al. 2013). The attractiveness of decision trees is due to the fact that, 390 decision trees represent rules. Rules can readily be expressed so that humans can 391 understand them (Berry and Linoff 2004). Decision trees provide the information 392 about which attribute alleles are most important for prediction or classification. As 393 shown in figure 3,4 and 5, different algorithms of decision trees were able to 394 produce the different decision trees with root and leaves. Random Forest model with 395 Accuracy criteria on Information Gain and Gini Index datasets were able to produce 396 decision trees with 100\% overall accuracy and precision (Fig. 4). The most 397 important attribute allele used to build this trees are bnlg1347a2 and bnlg381a3, 398 which can be used to visually and explicitly represent decisions and decision making 399 for susceptible and tolerance cultivars. Therefore, we are able to create a model that 400 predicts the value of a target variable by learning simple decision rules inferred from 401 the data features. It means simply by using 2 SSR markers, bnlg1347 and bnlg381, 402 drought tolerance inbred lines can be predictable.

403 Support Vector Machine are widely used in computational biology including 404 genomics, proteomics, metabonomics due to their high accuracy, their ability to deal 405 with high-dimensional datasets, and their flexibility in modeling diverse sources of 406 data (Cai and Jiang 2013, O'Fallon et al. 2013, Verma and Melcher 2012, Xie et al. 407 2009). SVM established two-class classification-based Machine Learning methods 408 can then be applied for developing an artificial intelligence system to classify a new 409 allele or fragment into the member or non-member class. Our results suggested that 410 only SVM Evolution algorithm with $100 \%$ overall accuracies and prediction (Table 6); 411 would be the best candidate algorithm to predict drought tolerance inbred lines if 412 they apply on Chi Square dataset.

413 Naïve Bayes based on Bayes conditional probability rule is used for 414 performing classification tasks. Naïve Bayes assumes the predictors are statistically 415 independent which makes it an effective classification tool that is easy to interpret 416 (Paoin 2011, Prabhakara and Acharya 2012). Two models, Naïve base (returns 
417 classification model using estimated normal distributions) and Naïve base kernel

418 (returns classification model using estimated kernel densities) used and the model

419 accuracy in predicting the right resistance - susceptible computed as stated before.

420 The need to accelerate breeding for better adaptation to drought and other

421 abiotic stresses is an issue of increasing urgency (Araus et al. 2008, Bänziger and

422 Cooper 2001, Ribaut et al. 2009). Hence, as traditional breeding appears to be

423 reaching a plateau; several approaches, which complement traditional with analytical

424 selection methodologies, may be required to further improvement (Araus, Slafer,

425 Royo and Serret 2008). The molecular approach has a great potential but actual

426 results and delivery towards water limited environments are meager. Although the

427 emergence new molecular techniques such as transcriptomics and proteomics

428 propose a revolutionary impact in analytical breeding, DNA marker technology is still

429 advantageous regarding cost/benefit and a potential partner for this recently

430 introduced discipline. Inter-disciplinary scientists have been trying to understand and

431 dissect the mechanisms of plant tolerance to drought stress using a variety of

432 approaches. Application fields such as molecular genetics, combined with increasing

433 computing power, supervised and unsupervised machine learning, can be used to

434 identify groups of alleles with similar patterns of expression, and this can help

435 provide answers to questions of how different alleles are affected by various traits

436 and which alleles are responsible for specific hereditary characters. We are able to

437 create models that have been applied successfully in the prediction, classification,

438 estimation, and pattern recognition in abiotic stress. The molecular genetics road

439 towards drought resistance is complex but we know that the destination is much

440 simpler. One of the objective of this article was to address the need to bring to the

441 molecular genetics community an increased understanding of knowledge discovery

442 from data so that these robust computing paradigms may be used even more

443 successfully in future molecular biology applications especially in abiotic and biotic

444 stress arias.

\section{Acknowledgement}

446 The author greatly appreciate support from department of Biology, Faculty of Science, 447 University of Qom. 


\section{References}

Araus J.L., Slafer G.A., Royo C., and Serret M.D. (2008) Breeding for yield potential and stress adaptation in cereals. Critical Reviews in Plant Science, 27:377412

Ashrafi E., Alemzadeh A., Ebrahimi M., Ebrahimie E., and Dadkhodaei N. (2011) Amino Acid Features of P1B-ATPase Heavy Metal Transporters Enabling Small Numbers of Organisms to Cope with Heavy Metal Pollution. Bioinformics and Biology Insights, 5:59-82.

Bänziger M., and Cooper M. (2001) Breeding for low input conditions and consequences for participatory plant breeding examples from tropical maize and wheat. Euphytica ,122:503-519

Baumgartner C., Lewis G.D., Netzer M., Pfeifer B., and Gerszten R.E. (2010) A new data mining approach for profiling and categorizing kinetic patterns of metabolic biomarkers after myocardial injury. Bioinformatics, 26:1745-1751.

Beiki A.H, Saboor S., and Ebrahimi M. (2012) A New Avenue for Classification and Prediction of Olive Cultivars Using Supervised and Unsupervised Algorithms. PLoS One, 7:e44164

Benesova M., Hola D., Fischer L., Jedelsky P.L., Hnilicka F., Wilhelmova N., Rothova O., Kocova M., Prochazkova D., Honnerova J., Fridrichova L., and Hnilickova $\mathrm{H}$. (2012) The physiology and proteomics of drought tolerance in maize: early stomatal closure as a cause of lower tolerance to short-term dehydration? PloS one, 7:e38017.

Berry M.J., and Linoff G.S. (2004) Data Mining Techniques: For Marketing, Sales, and Customer Relationship Management. New York .Wiley.

Blum A., (2011) Drought resistance - is it really a complex trait? Functional Plant Biology, 38:753-757.

Bravo A., and Medina R. (2008) An unsupervised clustering framework for automatic segmentation of left ventricle cavity in human heart angiograms. Computerized medical imaging and graphics. the official journal of the Computerized Medical Imaging Society, 32:396-408. 
Cai B., and Jiang X. (2013) A novel artificial neural network method for biomedical prediction based on matrix pseudo-inversion. The Journal of Biomedical Informatics, 48:114-21.

Casanova J.J., O'Shaughnessy S.A., Evert S.R., Rush C.M. (2014) Development of a wireless computer vision instrument to detect biotic stress in wheat. Sensors (Basel), 14(9): 17753-17769.

Daryanto S, Wang L, Jacinthe P-A (2016) Global Synthesis of Drought Effects on Maize and Wheat Production. PLoS ONE 11(5): e0156362

Dror G., Sorek R., and Shamir R. (2005) Accurate identification of alternatively spliced exons using support vector machine. Bioinformatics, 21:897-901.

Ebrahimi M., Lakizadeh A., Agha-Golzadeh P., and Ebrahimie E. (2011) Prediction of thermostability from amino acid attributes by combination of clustering with attribute weighting: a new vista in engineering enzymes. PLoS One, 6:e23146.

El Harchaoui N.E., Ait Kerroum M., Hammouch A., Ouadou M., and Aboutajdine D. (2013) Unsupervised approach data analysis based on fuzzy possibilistic clustering: application to medical image MRI. Computational intelligence and neuroscience, 3:435497.

Ercisli S., Ipek A., and Barut E. (2011) SSR marker-based DNA fingerprinting and cultivar identification of olives (Olea europaea). Biochemical genetics, 49:555561.

Fleury D, Jefferies S, Kuchel H, Langridge P (2010) Genetic and genomic tools to improve drought tolerance in wheat. Journal of experimental botany, 61:32113222.

Forghani Y., and Yazdi H.S. (2014) Robust support vector machine-trained fuzzy system. Neural networks. the official journal of the International Neural Network Society, 50:154-165.

Garcia-Gonzalez D.L., Romero N., and Aparicio R. (2010) Comparative study of virgin olive oil quality from single varieties cultivated in Chile and Spain. Journal of Agricultural and Food Chemistry, 58:12899-12905. 
Hajiloo M., Rabiee H.R., and Anooshahpour M. (2013) Fuzzy support vector machine: an efficient rule-based classification technique for microarrays. BMC bioinformatics, 14 Suppl 13:S4.

Handfield L.F., Chong Y.T., Simmons J., Andrews B.J., and Moses A.M. (2013) Unsupervised clustering of subcellular protein expression patterns in highthroughput microscopy images reveals protein complexes and functional relationships between proteins. PLoS computational biology 9(6):e1003085.

Hepworth P.J., Nefedov A.V., Muchnik I.B., and Morgan K.L. (2012) Broiler chickens can benefit from machine learning: support vector machine analysis of observational epidemiological data. Journal of the Royal Society Interface, 9:1934-1942.

Hor C.Y., Yang C.B., Yang Z.J., and Tseng C.T. (2013) Prediction of protein essentiality by the support vector machine with statistical tests. Evolutionary bioinformatics online, 9:387-416.

Khazaei H., Street K., Bari A., Mackay M., and Stoddard F.L. (2013) The FIGS (focused identification of germplasm strategy) approach identifies traits related to drought adaptation in Vicia faba genetic resources. PLOS ONE, 8, e63107.

Kuska M., Wahabzada M., Leucker M., Dehne H.W., Kersting K., Oerke E.C., Steiner E., and Mahlein A.K. (2015) Hyperspectral phenotyping on the microscopic scale: towards automated characterization of plant-pathogen interactions. Plant Methods, 15: 11, 28.

Liu X., Zhai S., Zhao Y., Sun B., Liu C., Yang A., and Zhang J. (2013) Overexpression of the phosphatidylinositol synthase gene (ZmPIS) conferring drought stress tolerance by altering membrane lipid composition and increasing ABA synthesis in maize. Plant, cell and environment, 36(5):10371055.

Lobell D.B., Roberts M.J., Schlender W., Braun N., Little B.B., Rejesus M.R., and Hammer G.L. (2014) Greater sensitivity to drought accompanies maize yield increase in the U.S. Midwest. Science, 344, 516-519. 
Ma H., Chang W., and Cui G. (2012) Ecological footprint model using the support vector machine technique. PloS one, 7:e30396.

Maenhout S., De Baets B., and Haesaert G. (2010) Prediction of maize single-cross hybrid performance: support vector machine regression versus best linear prediction. Theoretical and Applied Genetics, 120:415-427.

Maenhout S., De Baets B., Haesaert G., and Van Bockstaele E. (2007) Support vector machine regression for the prediction of maize hybrid performance. Theoretical and Applied Genetics, 115:1003-1013.

Markovich Gordon M., Moser A.M., and Rubin E. (2012) Unsupervised analysis of classical biomedical markers: robustness and medical relevance of patient clustering using bioinformatics tools. PloS one, 7:e29578.

Migliorini M., Mariani S., Bianchi A.M. (2013) Decision tree for smart feature extraction from sleep $\mathrm{HR}$ in bipolar patients. Conference of the IEEE Engineering in Medicine and Biology Society, 2013:5033-5036.

Mir R.R., Zaman-Allah M., Sreenivasulu N., Trethowan R., and Varshney R.K. (2012) Integrated genomics, physiology and breeding approaches for improving drought tolerance in crops. Theoretical and Applied Genetics, 125:625-645.

Mutka A., and Bart R., (2015) Image-based phenotyping of plant disease symptoms. Frontiers in plant science, 5; 5: 734.

O'Fallon B.D., Wooderchak-Donahue W., and Crockett D.K. (2013) A support vector machine for identification of single-nucleotide polymorphisms from nextgeneration sequencing data. Bioinformatics, 29:1361-1366.

Ornella L., Cervigni G., and Tapia E. (2012) Applications of Machine Learning in Breeding for Stress Tolerance in MaizeCrop Stress and its Management: Perspectives and Strategies. Springer, pp. 163-192.

Pafundo S., Agrimonti C., and Marmiroli N. (2005) Traceability of plant contribution in olive oil by amplified fragment length polymorphisms. Journal of agricultural and food chemistry, 53:6995-7002.

Paoin W. (2011) Lessons learned from data mining of WHO mortality database. Methods of information in medicine, 50:380-385. 
Prabhakara S., and Acharya R. (2012) Unsupervised two-way clustering of metagenomic sequences. Journal of biomedicine and biotechnology, 153647.

Prasanna B.M., Beiki A.H., Sekhar J.C., Srinivas A., and Ribaut J.M. (2009) Mapping QTLs for component traits influencing drought stress tolerance of maize. Indian Journal of Plant Biochemistry and Biotechnology, 18:151-160.

Ravi K., Vadez V., Isobe S., Mir R.R., Guo Y., Nigam S.N., Gowda M.V., Radhakrishnan T., Bertioli D.J., Knapp S.J., and Varshney R.K. (2011) Identification of several small main-effect QTLs and a large number of epistatic QTLs for drought tolerance related traits in groundnut (Arachis hypogaea L.). Theoretical and Applied Genetics, 122:1119-1132.

Reale S., Doveri S., Diaz A., Angiolillo A., Lucentini L., Pilla F., Martin A., Donini P., and Lee D. (2006) SNP-based markers for discriminating olive (Olea europaea L.) cultivars. Genome, 49:1193-1205.

Ribaut J-M, Betran J., Monneveux P., and Setter T. (2009) Drought Tolerance in Maize. In: Bennetzen J, Hake S (eds) Handbook of Maize: Its Biology. Springer New York, pp. 311-344.

Römer C., Wahabzada M., Ballvora A., Pinto F., Rossini M., Cinzia P., Behmann J., Leon J., Thurau C., Bauckhage C., Kersting K., Rashcher U., and Pluner L. (2009) Early drought stress detection in cereals: simplex volume maximisation for hyperspectra image analysis. Functinal Plant Biology, 39, 878.

Senthil Kumar A.R., Goyal M.K., Ojha C.S., Singh R.D., and Swamee P.K. (2013) Application of artificial neural network, fuzzy logic and decision tree algorithms for modelling of streamflow at Kasol in India. Water Science Technology, 68:2521-2526.

Shinozaki K., and Yamaguchi-Shinozaki K. (2007) Gene networks involved in drought stress response and tolerance. Journal of experimental botany, 58:221-227.

Smith P.F., Haslett S., and Zheng Y. (2013) A multivariate statistical and data mining analysis of spatial memory-related behaviour following bilateral vestibular loss in the rat. Behavioural brain research, 246:15-23. 
Tao Z., Kou Y., Liu H., Li X., Xiao J., and Wang S. (2011) OsWRKY45 alleles play different roles in abscisic acid signalling and salt stress tolerance but similar roles in drought and cold tolerance in rice. Journal of experimental botany, 62:4863-4874.

Tardieu F. (2012) Any trait or trait-related allele can confer drought tolerance: just design the right drought scenario. Journal of experimental botany, 63:25-31.

Tran L. (2014) The Un-normalized Graph p-Laplacian Based Semi-supervised Learning Method and Protein Function Prediction Problem Knowledge and Systems Engineering, Springer.

Verma R., and Melcher U. (2012) A Support Vector Machine based method to distinguish proteobacterial proteins from eukaryotic plant proteins. BMC bioinformatics, 13 Suppl 15:S9.

Wang W.S., Pan Y.J., Zhao X.Q., Dwivedi D., Zhu L.H., Ali J., Fu B.Y., and Li Z.K. (2011) Drought-induced site-specific DNA methylation and its association with drought tolerance in rice (Oryza sativa L.). Journal of experimental botany, 62:1951-1960.

Wang X., Wang H., Liu S., Ferjani A., Li J., Yan J., and Yang X . (2016). Genetic Variation in ZmVPP1 contributes to drought tolerance im maize seedlings . Nature Genetics , 48:1233-12141.

Wang Y., and Tseng M. M. (2013). A Naïve Bayes approach to map customer requirements to product variants. Journal of Intelligent Manufacturing, 26: 501-509.

Xie H.B., Zheng Y.P., Guo J.Y., Chen X., and Shi J. (2009) Estimation of wrist angle from sonomyography using support vector machine and artificial neural network models. Medical engineering and physics, 31:384-391.

Xu Y., Skinner D.J., Wu H., Palacios-Rojas N., Araus J.L., Yan J., Gao S., Warburton M.L., and Crouch J.H. (2009). Advances in maize genomics and their value for enhancing genetic gains from breeding. International Journal of Plant Genomics, 2009:957602. 
Yadav R.S., Sehgal D., and Vadez V. (2011) Using genetic mapping and genomics approaches in understanding and improving drought tolerance in pearl millet. Journal of experimental botany, 62:397-408.

Yan A., Hu X., Wang K., and Sun J. (2013) Discriminating of ATP competitive Src kinase inhibitors and decoys using self-organizing map and support vector machine. Molecular diversity, 17:75-83.

Yu C. (2011) China's water crisis needs more than words. Nature, 470, 307.

Yun Y.E., Cotton C.A., and Edginton A.N. (2014) Development of a decision tree to classify the most accurate tissue-specific tissue to plasma partition coefficient algorithm for a given compound. Journal of Pharmacokinet Pharmacodyn, 41:1-14.

Figure Legend List 
Figure 1 SSR fragment alleles can often be distinguished on agarose gelsError! Bookmark not defined.

Figure 2 Application of the SVC algorithm on ten datasets (A) was unable to categorize inbred lines into correct clusters; K- Medoids algorithm to the Deviation $(B)$ and to PCA(C) was able to categorize drought tolerance inbred lines into correct clusters and K- means algorithm to the PCA (C) was able to categorize susceptible tolerance inbred lines into correct clustersError! Bookmark not defined.

Figure 3 Almost Decision Stump, Decision Tree and Decision Parallel in 4 criteria gain ratio, information gain, gini index and accuracy on all databases can produce the same simple decision tree

Error! Bookmark not defined.

Figure 4 Decision trees on Random Forest model with Accuracy criterion run on Information Gain dataset (A ) and Gini Index Criterion run on Gini Index dataset (B)Error! Bookmark not defined.

Figure 5 Random Forest model with Chi Square dataset when run on Accuracy (A), Gain Ratio (B) and Gini Index criteria (C); and with Information Gain dataset on Gini Index criterion (D). Error!

Bookmark not defined.

Table1- Name, repeat type and bin location of SSR marker

\begin{tabular}{lcclcccccc}
\hline $\begin{array}{c}\text { SSR } \\
\text { Locus }\end{array}$ & $\begin{array}{c}\text { Repeat } \\
\text { Type }\end{array}$ & $\begin{array}{c}\text { Bin } \\
\text { Location }\end{array}$ & $\begin{array}{c}\text { SSR } \\
\text { Locus }\end{array}$ & $\begin{array}{c}\text { Repeat } \\
\text { Type }\end{array}$ & $\begin{array}{c}\text { Bin } \\
\text { Location }\end{array}$ & $\begin{array}{c}\text { SSR } \\
\text { Locus }\end{array}$ & $\begin{array}{c}\text { Repeat } \\
\text { Type }\end{array}$ & $\begin{array}{c}\text { Bin } \\
\text { Location }\end{array}$ \\
\hline phi097 & TAG & 1.01 & bnlg420 & CT & 3.05 & phi089 & ATGC & 6.08 \\
\hline bnlg147 & CA & 1.02 & phi088 & ACT & 3.08 & bnlg339 & CT & 7.03 \\
\hline bnlg1016 & AG & 1.04 & bnlg1182 & AG & 3.09 & bnlg155 & CT & 7.04 \\
\hline bnlg1556 & AG & 1.06 & bnlg2244 & AG & 4.03 & phi116 & AGTG-ACG & 7.06 \\
\hline phi037 & AG & 1.08 & bnlg1729 & AG & 4.05 & phi119 & AG & 8.02 \\
\hline bnlg400 & AG & 1.09 & bnlg252 & AG & 4.06 & phi014 & GGC & 8.04 \\
\hline bnlg1347 & AG & 1.1 & bnlg589 & AG & 4.11 & bnlg162 & CA & 8.05 \\
\hline bnlg504 & CA & 1.11 & phi113 & GTCT & 5.02 & bnlg240 & AG & 8.06 \\
\hline umc1331 & GGT & 1.11 & bnlg2323 & AG & 5.04 & bnlg1065 & AG & 8.06 \\
\hline bnlg1092 & AG & 2.01 & phi101 & ACT & 5.06 & phi015 & AAAC & $8.08-8.09$ \\
\hline bnlg2042 & AG & 2.02 & bnlg389 & CT & 5.09 & phi080 & AGGAG & $8.08-8.09$ \\
\hline bnlg1297 & AG & 2.02 & bnlg1043 & AG & 6 & bnlg1272 & AG & 9 \\
\hline bnlg1064 & AG & 2.03 & phi077 & AG & 6.01 & phi033 & AAG & 9.01 \\
\hline umc1555 & TTCA & 2.03 & umc1018 & CT & 6.01 & bnlg1730 & AG & 9.03 \\
\hline bnlg381 & CA & 2.03 & umc1006 & GA & 6.02 & bnlg1209 & AG & 9.05 \\
\hline bnlg1613 & AG & 2.04 & phi070 & AGCTG & 6.03 & phi041 & AGCC & 10 \\
\hline bnlg1175 & AG & 2.04 & umc1572 & GA & 6.03 & phi059 & ACC & 10.02 \\
\hline dup21 & AG & 2.05 & umc1014 & GA & 6.04 & bnlg210 & AG & 10.03 \\
\hline mage05 & AG & 2.05 & bnlg1154 & AG & 6.05 & bnlg1655 & AG & 10.03 \\
\hline
\end{tabular}




\begin{tabular}{|c|c|c|c|c|c|c|c|c|}
\hline bnlg121 & CT & 2.06 & bnlg1702 & $A G$ & 6.05 & bnlg1518 & $A G$ & 10.04 \\
\hline bnlg1138 & $A G$ & 2.06 & $\mathrm{mmc0241}$ & TA-N-TG & 6.05 & bnlg1526 & $A G$ & 10.04 \\
\hline bnlg1045 & $A G$ & 2.07 & phi078 & AAAG & 6.05 & bnlg594 & $A G$ & 10.06 \\
\hline bnlg198 & CT & 2.08 & phi102 & AT & 6.05 & phi035 & $A C$ & 10.06 \\
\hline bnlg1520 & $A G$ & 2.09 & nc013 & $A G$ & 6.05 & & & \\
\hline
\end{tabular}

Table 2- The number and the average of most important alleles (fragments) selected by different attribute weighting algorithms.

\begin{tabular}{lcc}
\hline $\begin{array}{l}\text { Alleles } \\
\text { (fragments) }\end{array}$ & $\begin{array}{l}\text { The number of } \\
\text { attribute } \\
\text { weightings }\end{array}$ & $\begin{array}{l}\text { The Average } \\
\text { of attribute } \\
\text { weightings }\end{array}$ \\
\hline phi033a3 & 9 & 0.91884 \\
bnlg1347a1 & 9 & 0.70050 \\
\hline bnlg1347a2 & 9 & 0.70050 \\
\hline bnlg172a2 & 9 & 0.69734 \\
\hline bnlg381a3 & 9 & 0.69649 \\
\hline phi078a2 & 9 & 0.59356 \\
\hline bnlg172a3 & 8 & 0.61950 \\
\hline umc1572a4 & 7 & 0.59898 \\
bnlg381a2 & 6 & 0.58188 \\
\hline
\end{tabular}


Table 3-The attribute weighting models and the numbers of important fragment alleles selected by each model and the most important variables selected by each attribute weighting algorithms.

\begin{tabular}{l|llllllll}
\hline $\begin{array}{l}\text { Attribute } \\
\text { Weighting }\end{array}$ & $\begin{array}{l}\text { Number } \\
\text { of } \\
\text { Variable }\end{array}$ & Important Atribute Fragment Alleles & & & \\
\hline Rule & 168 & phi033a3 & bnlg172a3 & phi078a2 & bnlg381a3 & bnlg172a2 & umc1572a4 \\
\hline Deviation & 147 & bnlg2323a1 & bnlg1730a4 & phi088a3 & bnlg1655a2 & mage05a3 & bnlg210a3 \\
\hline SVM & 133 & phi033a3 & bnlg1347a1 & bnlg1347a2 & bnlg172a2 & bnlg381a3 & bnlg1154a3 \\
\hline PCA & 92 & bnlg1138a1 & phi035a3 & bnlg18a3 & phi102a3 & phi102a2 & bnlg381a3 \\
\hline Uncertainty & 20 & phi033a3 & bnlg172a2 & bnlg1347a1 & bnlg1347a2 & umc1572a4 & bnlg172a3 \\
\hline InfoGainRatio & 20 & phi033a3 & bnlg172a2 & bnlg1347a1 & bnlg1347a2 & umc1572a4 & bnlg172a3 \\
\hline Gini Index & 8 & phi033a3 & bnlg172a2 & bnlg1347a1 & bnlg1347a2 & umc1572a4 & bnlg172a3 \\
\hline Relief & 8 & phi033a3 & bnlg172a2 & bnlg1347a1 & bnlg1347a2 & dup21a4 & bnlg252a2 \\
\hline Info Gain & 8 & phi033a3 & bnlg172a2 & bnlg1347a1 & bnlg1347a2 & umc1572a4 & bnlg172a3 \\
\hline Chi Squared & 7 & phi033a3 & bnlg172a2 & bnlg1347a1 & bnlg1347a2 & umc1572a4 & bnlg172a3 \\
\hline
\end{tabular}


bioRxiv preprint doi: https:/doi.org/10 1101/578880; this version posted March 16, 2019. The copyright holder for this preprint (which was not certified by peer review) is the author/funder, who has granted bioRxiv a license to display the preprint in perpetuity. It is made available under aCC-BY 4.0 International license.

Table 4- Percent correct class clustering of the K-Means and K- Medoids to SVM, PCA and

Deviation databases

\begin{tabular}{llrr}
\hline Unsupervised Algorithm & Dataset & Susceptible & \multicolumn{1}{c}{ Tolerance } \\
\hline K-Means & SVM & 33.3 & 66.7 \\
& PCA & 83.3 & 33.3 \\
& Deviation & 66.7 & 50.0 \\
K-Medoids & SVM & 66.7 & 50.0 \\
& PCA & 50.0 & 83.3 \\
& Deviation & 50.0 & 100.0 \\
\hline
\end{tabular}


Table 5- Overall Accuracy and Precision; Precision and Recall Tolerance and Susceptible in Random forest with 4 criteria run on Chi Squre, Deviation, Information Gain and Gini Index Datasets in decision trees

\begin{tabular}{|c|c|c|c|c|c|c|c|}
\hline 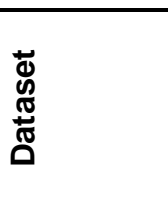 & $\frac{\pi}{\stackrel{\frac{\pi}{2}}{\frac{\pi}{2}}}$ & 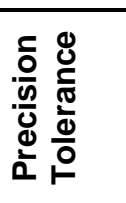 & 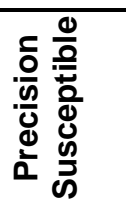 & 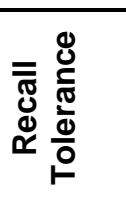 & 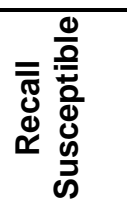 & 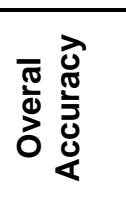 & 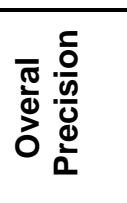 \\
\hline \multirow[t]{4}{*}{ Chi Squre } & Accuracy & 86.0 & 100.0 & 100.0 & 83.3 & 90.0 & 100.0 \\
\hline & Gain ratio & 86.0 & 100.0 & 100.0 & 83.3 & 90.0 & 100.0 \\
\hline & Gini Index & 86.0 & 100.0 & 100.0 & 83.3 & 90.0 & 100.0 \\
\hline & Info. Gain & 75.0 & 100.0 & 100.0 & 66.7 & 85.0 & 100.0 \\
\hline \multirow[t]{4}{*}{ Deviation } & Accuracy & 57.0 & 60.0 & 67.0 & 50.0 & 65.0 & 60.0 \\
\hline & Gain ratio & 50.0 & 50.0 & 67.0 & 33.0 & 45.0 & 50.0 \\
\hline & Gini Index & 80.0 & 71.0 & 67.0 & 83.0 & 75.0 & 71.0 \\
\hline & Info. Gain & 33.0 & 0.00 & 50.0 & 0.00 & 25.0 & 0.00 \\
\hline \multirow[t]{4}{*}{ Info. Gain } & Accuracy & 100 & 100.0 & 100 & 100.0 & 100.0 & 100.0 \\
\hline & Gain ratio & 75.0 & 100.0 & 100.0 & 67.0 & 80.0 & 100.0 \\
\hline & Gini Index & 86.0 & 100.0 & 100.0 & 83.0 & 90.0 & 100.0 \\
\hline & Info. Gain & 75.0 & 100.0 & 100.0 & 67.0 & 85.0 & 100.0 \\
\hline \multirow[t]{4}{*}{ Gini Index } & Accuracy & 75.0 & 100.0 & 100.0 & 67.0 & 80.0 & 100.0 \\
\hline & Gain ratio & 67.0 & 100.0 & 100.0 & 50.0 & 70.0 & 100.0 \\
\hline & Gini Index & 100.0 & 100.0 & 100.0 & 100.0 & 100.0 & 100.0 \\
\hline & Info. Gain & 71.0 & 80.0 & 83.0 & 67.0 & 70.0 & 80.0 \\
\hline
\end{tabular}


Table 6- The accuracy, precision and recall of different models of SVM with different databases

\begin{tabular}{|c|c|c|c|c|c|c|c|}
\hline Dataset & algorithm & 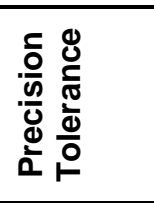 & 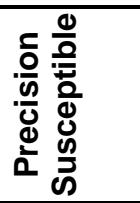 & 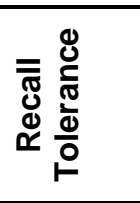 & 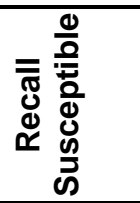 & 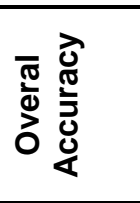 & 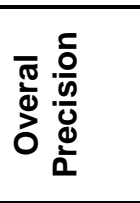 \\
\hline \multirow[t]{4}{*}{ Chi Square } & Evolution & 100 & 100 & 100 & 100 & 100 & 100 \\
\hline & LibSVM & 85 & 100 & 100 & 83 & 90 & 100 \\
\hline & SVMLinear & 85 & 100 & 100 & 83 & 90 & 100 \\
\hline & SVM & 85 & 100 & 100 & 83 & 90 & 100 \\
\hline \multirow[t]{4}{*}{ Deviation } & Evolution & 50 & 50 & 50 & 50 & 45 & 50 \\
\hline & LibSVM & 0 & 14 & 0 & 17 & 5 & 14 \\
\hline & SVMLinear & 50 & 50 & 83 & 17 & 50 & 50 \\
\hline & SVM & 50 & 50 & 83 & 17 & 50 & 50 \\
\hline \multirow[t]{4}{*}{ FCdb } & Evolution & 25 & 37 & 17 & 50 & 35 & 35 \\
\hline & LibSVM & 0 & 14 & 0 & 17 & 5 & 14 \\
\hline & SVMLinear & 50 & 50 & 83 & 17 & 50 & 50 \\
\hline & SVM & 50 & 50 & 83 & 17 & 50 & 50 \\
\hline \multirow[t]{4}{*}{ Gini Index } & Evolution & 100 & 100 & 100 & 100 & 100 & 100 \\
\hline & LibSVM & 85 & 100 & 100 & 83 & 90 & 100 \\
\hline & SVMLinear & 85 & 100 & 100 & 83 & 90 & 100 \\
\hline & SVM & 85 & 100 & 100 & 83 & 90 & 100 \\
\hline Inf.Gain & Evolution & 85 & 100 & 100 & 83 & 90 & 100 \\
\hline \multirow[t]{3}{*}{ Ratio } & LibSVM & 100 & 100 & 100 & 100 & 100 & 100 \\
\hline & SVMLinear & 86 & 100 & 100 & 83 & 95 & 100 \\
\hline & SVM & 86 & 100 & 100 & 83 & 95 & 100 \\
\hline \multirow[t]{3}{*}{ Info. Gain } & Evolution & 100 & 100 & 100 & 100 & 100 & 100 \\
\hline & LibSVM & 86 & 100 & 100 & 83 & 95 & 100 \\
\hline & SVMLinear & 86 & 100 & 100 & 83 & 95 & 100 \\
\hline
\end{tabular}




\begin{tabular}{|c|c|c|c|c|c|c|c|}
\hline & SVM & 86 & 100 & 100 & 83 & 95 & 100 \\
\hline \multirow[t]{4}{*}{ PCA } & Evolution & 50 & 50 & 33 & 67 & 45 & 50 \\
\hline & LibSVM & 0 & 0 & 0 & 0 & 0 & 0 \\
\hline & SVMLinear & 55 & 67 & 83 & 33 & 60 & 67 \\
\hline & SVM & 55 & 67 & 83 & 33 & 60 & 67 \\
\hline \multirow[t]{4}{*}{ Relief } & Evolution & 100 & 100 & 100 & 100 & 100 & 100 \\
\hline & LibSVM & 86 & 100 & 100 & 83 & 95 & 100 \\
\hline & SVMLinear & 86 & 100 & 100 & 83 & 95 & 100 \\
\hline & SVM & 86 & 100 & 100 & 83 & 95 & 100 \\
\hline \multirow[t]{4}{*}{ Rule } & Evolution & 37 & 25 & 50 & 17 & 35 & 25 \\
\hline & LibSVM & 17 & 17 & 17 & 17 & 17 & 17 \\
\hline & SVMLinear & 71 & 80 & 83 & 67 & 70 & 80 \\
\hline & SVM & 71 & 80 & 83 & 67 & 70 & 80 \\
\hline \multirow[t]{4}{*}{ SVM } & Evolution & 50 & 50 & 50 & 50 & 50 & 50 \\
\hline & LibSVM & 100 & 86 & 83 & 100 & 90 & 100 \\
\hline & SVMLinear & 75 & 100 & 100 & 67 & 85 & 100 \\
\hline & SVM & 75 & 100 & 100 & 67 & 85 & 100 \\
\hline \multirow[t]{4}{*}{ Uncertainty } & Evolution & 100 & 100 & 100 & 100 & 100 & 100 \\
\hline & LibSVM & 100 & 100 & 100 & 100 & 100 & 100 \\
\hline & SVMLinear & 86 & 100 & 100 & 83 & 95 & 100 \\
\hline & SVM & 86 & 100 & 100 & 83 & 95 & 100 \\
\hline
\end{tabular}

Table 7-The accuracy, precision and recall of Naïve Bayse and Naïve kernel Models with different databases

\begin{tabular}{|c|c|c|c|c|c|c|c|}
\hline Dataset & Model & 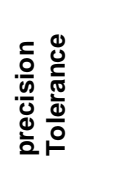 & 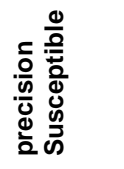 & 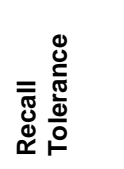 & 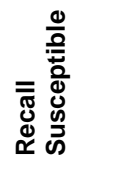 & 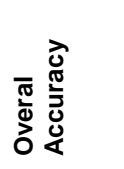 & 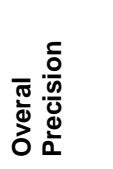 \\
\hline \multirow[t]{2}{*}{ Chi Squared } & naïve Byase & $85.71 \%$ & $100.00 \%$ & $100.00 \%$ & $83.33 \%$ & $90.00 \%$ & $100.00 \%$ \\
\hline & naïve Karnel & $85.71 \%$ & $100.00 \%$ & $100.00 \%$ & $83.33 \%$ & $90.00 \%$ & $100.00 \%$ \\
\hline \multirow[t]{2}{*}{ Deviation } & naïve Byase & $16.67 \%$ & $16.67 \%$ & $16.67 \%$ & $16.67 \%$ & $15.00 \%$ & $16.67 \%$ \\
\hline & naïve Karnel & $62.50 \%$ & $75.00 \%$ & $83.33 \%$ & $50.00 \%$ & $65.00 \%$ & $75.00 \%$ \\
\hline \multirow[t]{2}{*}{ FC data } & naïve Byase & $62.50 \%$ & $75.00 \%$ & $83.33 \%$ & $50.00 \%$ & $65.00 \%$ & $75.00 \%$ \\
\hline & naïve Karnel & $62.50 \%$ & $75.00 \%$ & $83.33 \%$ & $50.00 \%$ & $65.00 \%$ & $75.00 \%$ \\
\hline \multirow[t]{2}{*}{ Gini Index } & naïve Byase & $100.00 \%$ & $100.00 \%$ & $100.00 \%$ & $100.00 \%$ & $100.00 \%$ & $100.00 \%$ \\
\hline & naïve Karnel & $100.00 \%$ & $100.00 \%$ & $100.00 \%$ & $100.00 \%$ & $100.00 \%$ & $100.00 \%$ \\
\hline \multirow[t]{2}{*}{ Info Gain } & naïve Byase & $100.00 \%$ & $100.00 \%$ & $100.00 \%$ & $100.00 \%$ & $100.00 \%$ & $100.00 \%$ \\
\hline & naïve Karnel & $100.00 \%$ & $100.00 \%$ & $100.00 \%$ & $100.00 \%$ & $100.00 \%$ & $100.00 \%$ \\
\hline \multirow[t]{2}{*}{ Ino Gain Ratio } & naïve Byase & $100.00 \%$ & $100.00 \%$ & $100.00 \%$ & $100.00 \%$ & $100.00 \%$ & $100.00 \%$ \\
\hline & naïve Karnel & $100.00 \%$ & $100.00 \%$ & $100.00 \%$ & $100.00 \%$ & $100.00 \%$ & $100.00 \%$ \\
\hline \multirow[t]{2}{*}{ PCA } & naïve Byase & $40.00 \%$ & $42.86 \%$ & $33.33 \%$ & $50.00 \%$ & $40.00 \%$ & $42.80 \%$ \\
\hline & naïve Karnel & $40.00 \%$ & $42.86 \%$ & $33.33 \%$ & $50.00 \%$ & $40.00 \%$ & $42.80 \%$ \\
\hline \multirow[t]{2}{*}{ Relif } & naïve Byase & $100.00 \%$ & $100.00 \%$ & $100.00 \%$ & $100.00 \%$ & $100.00 \%$ & $100.00 \%$ \\
\hline & naïve Karnel & $100.00 \%$ & $100.00 \%$ & $100.00 \%$ & $100.00 \%$ & $100.00 \%$ & $100.00 \%$ \\
\hline \multirow[t]{2}{*}{ Rule } & naïve Byase & $83.30 \%$ & $83.30 \%$ & $83.30 \%$ & $83.30 \%$ & $80.00 \%$ & $83.30 \%$ \\
\hline & naïve Karnel & $83.30 \%$ & $83.30 \%$ & $83.30 \%$ & $83.30 \%$ & $80.00 \%$ & $83.30 \%$ \\
\hline \multirow[t]{2}{*}{ SVM } & naïve Byase & $100.00 \%$ & $100.00 \%$ & $100.00 \%$ & $100.00 \%$ & $100.00 \%$ & $100.00 \%$ \\
\hline & naïve Karnel & $100.00 \%$ & $100.00 \%$ & $100.00 \%$ & $100.00 \%$ & $100.00 \%$ & $100.00 \%$ \\
\hline Uncertainty & naïve Byase & $100.00 \%$ & $100.00 \%$ & $100.00 \%$ & $100.00 \%$ & $100.00 \%$ & $100.00 \%$ \\
\hline
\end{tabular}


bioRxiv preprint doi: https://doi.org/10.1101/578880; this version posted March 16,2019 . The copyright holder for this preprint (which was not certified by peer review) is the author/funder, who has granted bioRxiv a license to display the preprint in perpetuity. It is made available under aCC-BY 4.0 International license.

\begin{tabular}{rrrrrrr}
\hline naïve Karnel & $100.00 \%$ & $100.00 \%$ & $100.00 \%$ & $100.00 \%$ & $100.00 \%$ & $100.00 \%$ \\
\hline
\end{tabular}

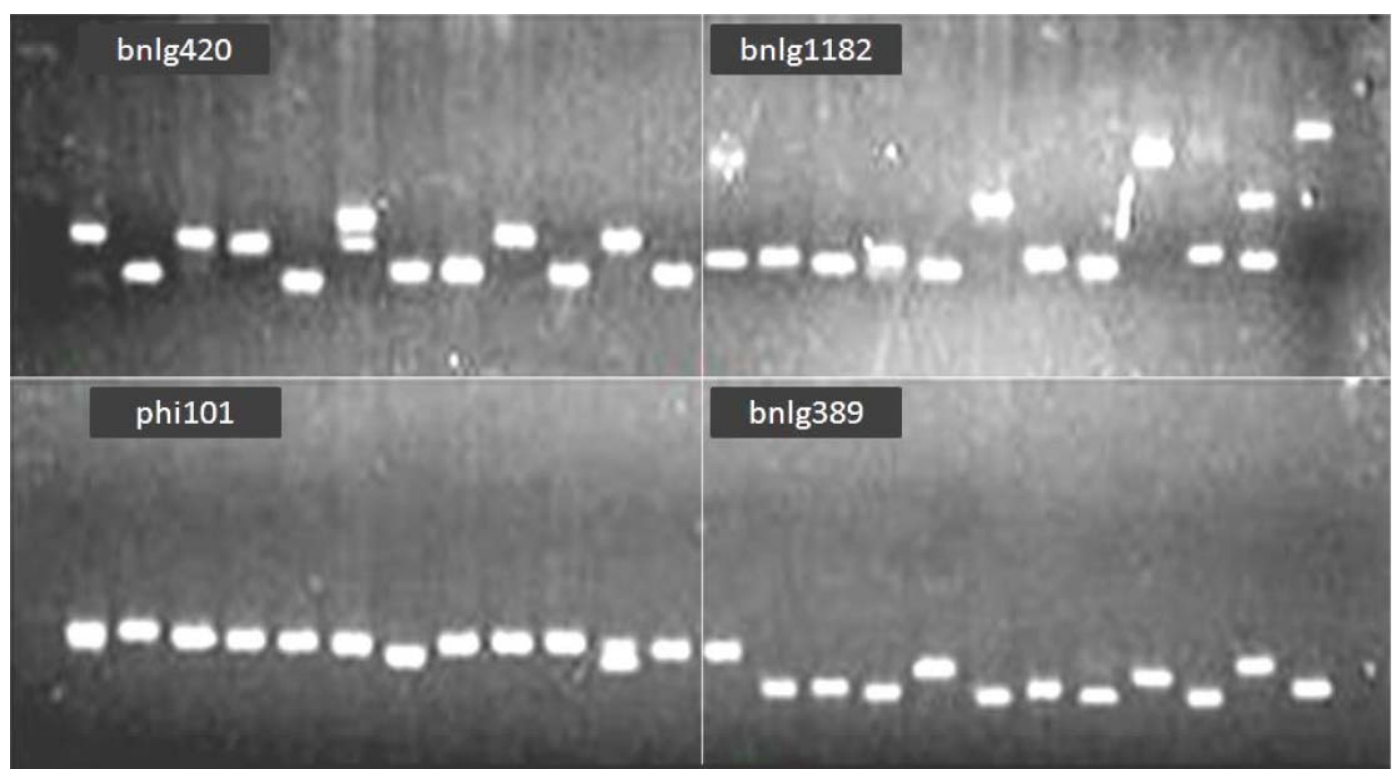

Figure 1- SSR fragment alleles can often be distinguished on agarose gels 
bioRxiv preprint doi: https://doi.org/10.1101/578880; this version posted March 16, 2019. The copyright holder for this preprint (which was not certified by peer review) is the author/funder, who has granted bioRxiv a license to display the preprint in perpetuity. It is made available under aCC-BY 4.0 International license.

A

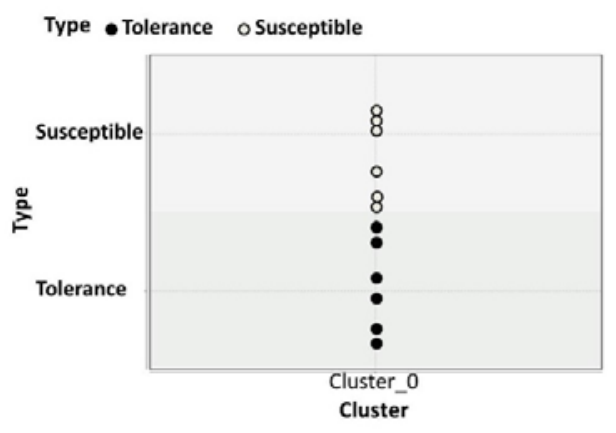

C

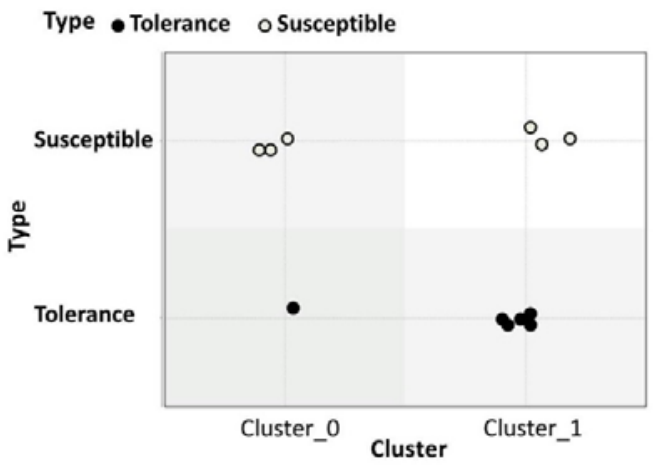

B

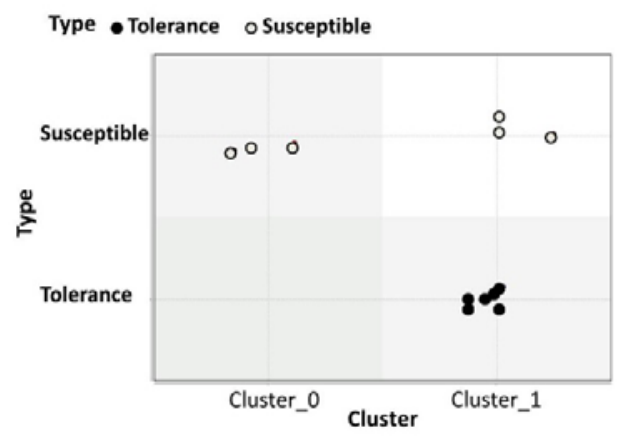

D

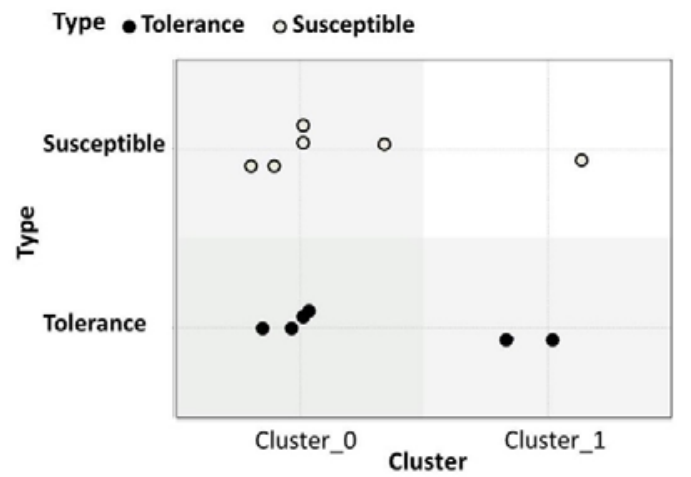

Figure 2- Application of the SVC algorithm on ten datasets (A) was unable to categorize inbred lines into correct clusters; K- Medoids algorithm to the Deviation $(\mathrm{B})$ and to PCA(C) was able to categorize drought tolerance inbred lines into correct clusters and K- means algorithm to the PCA (C) was able to categorize susceptible tolerance inbred lines into correct clusters 
bioRxiv preprint doi: https://doi.org/10.1101/578880; this version posted March 16, 2019. The copyright holder for this preprint (which was not certified by peer review) is the author/funder, who has granted bioRxiv a license to display the preprint in perpetuity. It is made available under aCC-BY 4.0 International license.

\section{phi033a3}

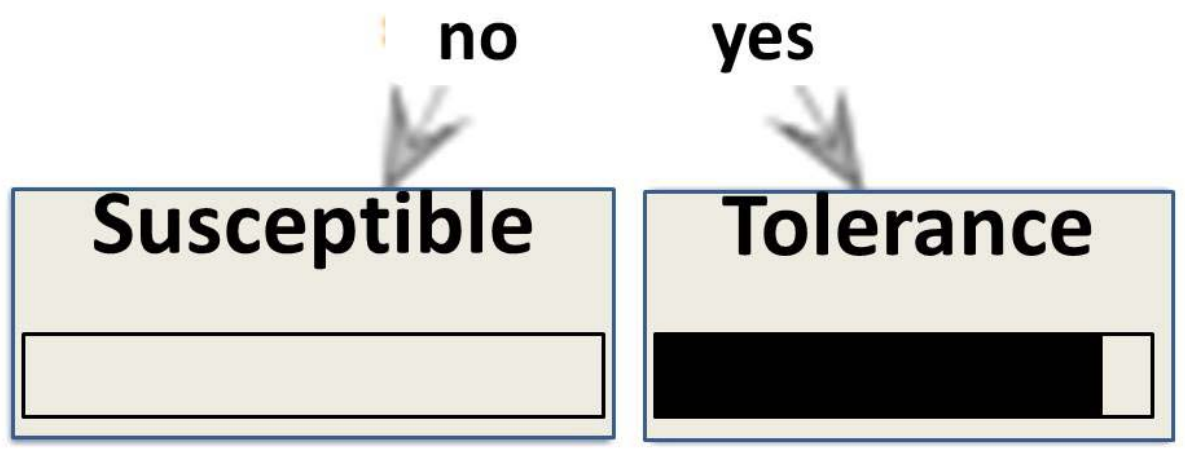

Figure 3- Almost Decision Stump, Decision Tree and Decision Parallel in 4 criteria gain ratio, information gain, gini index and accuracy on all databases can produce the same simple decision tree 
bioRxiv preprint doi: https://doi.org/10.1101/578880; this version posted March 16, 2019. The copyright holder for this preprint (which was not certified by peer review) is the author/funder, who has granted bioRxiv a license to display the preprint in perpetuity. It is made available under aCC-BY 4.0 International license.
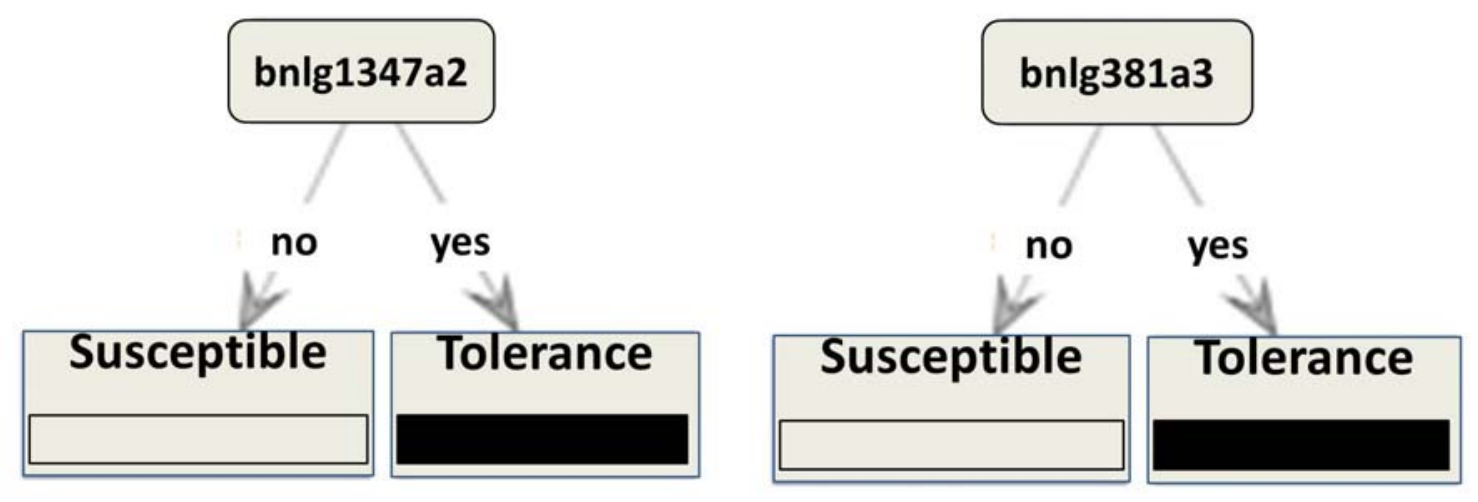

Figure 4- Decision trees on Random Forest model with Accuracy criterion run on Information Gain dataset (A) and Gini Index Criterion run on Gini Index dataset (B) 
A

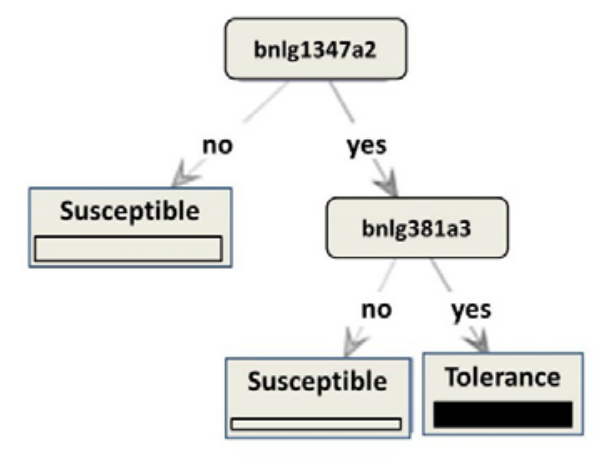

C

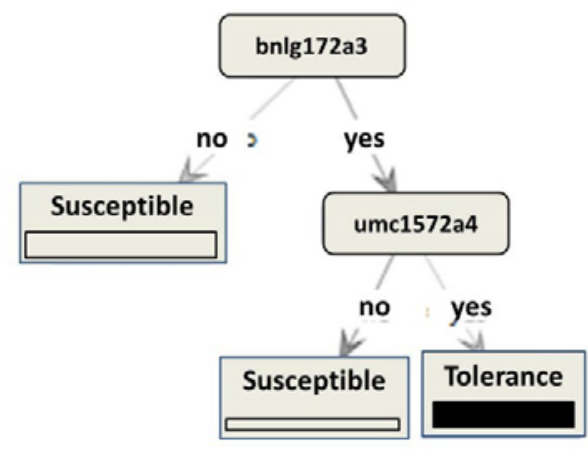

B

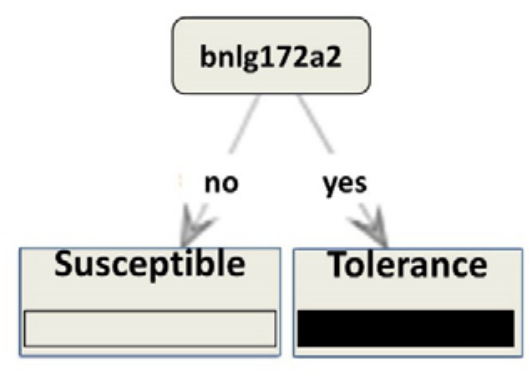

D

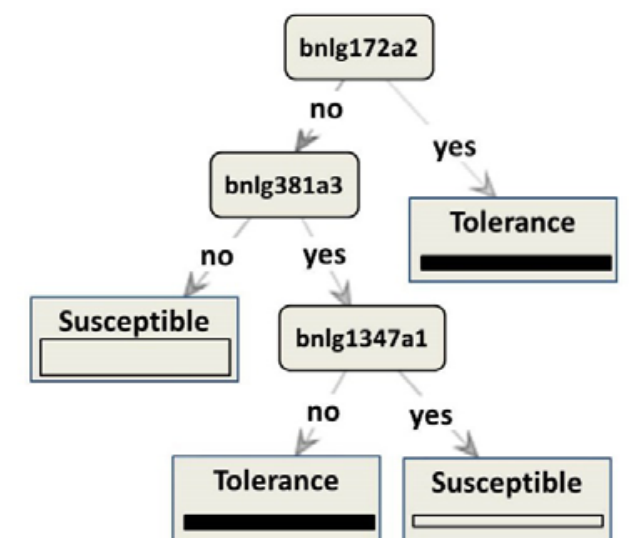

Figure 5- Random Forest model with Chi Square dataset when run on Accuracy (A), Gain Ratio (B) and Gini Index criteria (C); and with Information Gain dataset on Gini Index criterion (D). 